\title{
A faunistic and ecological study on Papilionoidea and Hesperioidea (Lepidoptera) of Sultandağları Mountains (Afyonkarahisar/Turkey)
}

\author{
Yusuf HÜSEYINOĞLU \\ Department of Biology, Faculty of Science and Letters, Mersin University, 33110 Mersin, Turkey.
}

Accepted 4 March, 2013

\begin{abstract}
In this study, the fauna of Papilionoidea and Hesperioidea (Lepidoptera) from Sultandağları Mountains, Afyonkarahisar province, in Turkey were investigated. Field collections were conducted between March and August from 1997 to 2001. Individuals were collected from 110 field surveys. The specimens belonged to 131 species in seven families, including Papilionidae (6 species), Pieridae (17), Libytheidae (1), Argynnidae (21), Satyridae (24), Lycaenidae (45), and Hesperiidae (17). Tomares nesimachus (Oberthür, 1893) was identified for the first time in Afyonkarahisar and fourteen of the 131 species were new records for the Lepidoptera fauna of Sultandağları Mountains.
\end{abstract}

Key words: Butterfly, fauna, Sultandağı, Çay, Sultandağları, biodiversity, Turkey.

\section{INTRODUCTION}

Today, certain species from Lepidoptera have partially become extinct or are under the risk of extinction due to the factors like environmental pollution in industrial countries, destruction of biotopes, effects of pesticides and the fact that some species have been collected for commercial purposes. Within the context of preserving biodiversity every country has to take the required conservation measures.

This study was conducted to contribute to identification of Sultandağları's diurnal Lepidoptera fauna, to define habitats of the species, to shed light on zoogeographic aspect of the fauna and finally to explore Afyonkarahisar province's biological richness.

The literature relating to Sultandağları's Lepidoptera fauna was reviewed from Koçak and Seven (1994). 30 scientific publications in total relating to the subject were reviewed. These faunistic studies include those conducted by Pfeiffer (1926-1927), Wagner (1929), and
Schwingenschuss $(1938,1939)$ in Sultandağları. Belter (1935) described a new subspecies from Brenthis daphne species collected in the region. Verity (19341936, 1935, 1936-1937, 1937-1938) recognized and named a couple of geographic variations from the species taking place in certain Satyridae and Lycaenidae families, which had been collected previously in Sultandağları, in his various articles. Lattin (1950) observed some butterfly species collected by Kosswig from various regions of Anatolia including Sultandağları and revised certain species, especially the species of Agrodiaetus, based on the material collected by Forster (1936, 1956, 1960, 1960-1961) and other various researchers and held in Munich Zoology Museum. In the study, various species and subspecies of Agrodiaetus living around Sultandağları and Akşehir were identified. Schurian and Rose (1991) identified a new subspecies from Lycaenidae species collected from Sultandağları. 
Table 1. Locations and dates of collections of butterflies in Sultandağı, Afyonkarahisar, Turkey. Locality Altitude Collection Date.

1: Yakasenek, Manastır $1400 \mathrm{~m} .13 .07 .1997$

2: Yakasenek, Manastır $1450 \mathrm{~m} .16 .05 .1999$

3: Yakasenek, Manastır 1450 m. 22.05.1999

4: Yakasenek, Manastır 1500 m. 24.06.2000

5: Yakasenek, Gelincikana $1750 \mathrm{~m} .13 .07 .1997$

6: Yakasenek, Gelincikana 1750 m. 17.07.1997

7: Yakasenek, Gelincikana 1700 m. 12.07.1998

8: Yakasenek, Gelincikana 1800 m. 12.07.1998

9: Yakasenek, Gelincikana 1750 m. 24.06.2000

10: Yakasenek, Gelincikana 1750 m. 24.06.2001

11: Yakasenek, Gelincikana 1860 m. 02.07.2000

12: Yakasenek, Gelincikana 1850 m. 14.07.2000

13: Yakasenek, Gelincikana 1900 m. 23.07.2000

14: Yakasenek, 1100-1200 m. 22.05.1999

15: Yakasenek $1300 \mathrm{~m}$. 04.07.1998

16: Yakasenek 1100-1250 m. 16.05.1999

17: Yakasenek $1200 \mathrm{~m}$. 14.04.2000

18: Yakasenek, Sarıpınar 1900 m. 23.07.2000

19: Yakasenek, Sütlüpınar 1500 m. 19.05.2000

20: Yakasenek, Sütlüpınar 1500 m. 24.06.2000

21: Yakasenek, Sütlüpınar 1400 m. 14.07.2000

22: Yakasenek, Sütlüpınar 1600 m. 23.07.2000

23: Yakasenek, Sütlüpınar 1450 m. 19.08 .2000

24: Yalama Hill 1400 m. 04.07.1998

25: Yalama Hill 1750 m. 04.07.1998

26: Yalama Hill 1200 m. 16.05.1999

27: Yalama Hill $1200 \mathrm{~m}$. 30.05.1999

28: Yalama Hill 1300 m. 30.05.1999

29: Yalama Hill 1500 m. 30.05.1999

30: Yalama Hill 1200 m. 16.08.1999

31: Yalama Hill 1250 m. 19.05.2000

32: Yalama Hill 1200 m. 17.06.2000

33: Yalama Hill 1400 m. 17.06.2000

34: Yalama Hill 1500 m. 17.06.2000

35: Yalama Hill 1200 m. 02.07.2000

36: Yalama Hill, Borazan 1700 m. 12.07.1998

37: Yalama Hill, fire tower 1500 m. 11.07.1998

38: Yalama Hill, fire tower $1680 \mathrm{~m}$. 30.05 .1999

39: Yalama Hill, fire tower $1680 \mathrm{~m}$. 11.07.1999

40: Yalama Hill, fire tower $1700 \mathrm{~m}$. 17.06.2000

41: Yalama Hill, fire tower $1750 \mathrm{~m} .02 .07 .2000$

42: Dereçine S. $1200 \mathrm{~m}$. 20.06.1998

43: Dereçine S. $1400 \mathrm{~m}$. 20.06.1998

44: Dereçine S. 1300 m. 05.07.1998

Hesselbarth et al. (1995) reported the species identified in Sultandağları in their books on butterflies in Turkey.
Table 1 Contd.

\begin{tabular}{|l|}
\hline 45: Dereçine S. $1500 \mathrm{~m} .05 .07 .1998$ \\
\hline 46: Dereçine S. $1200 \mathrm{~m} .15 .05 .1999$ \\
\hline 47: Dereçine S. $1400 \mathrm{~m} .15 .05 .1999$ \\
\hline 48: Dereçine, $1100 \mathrm{~m} .18 .05 .1999$ \\
\hline 49: Dereçine, $1290 \mathrm{~m} .15 .05 .1999$ \\
\hline 50: Dereçine, $1280 \mathrm{~m} .13 .05 .2000$ \\
\hline 51: Dereçine, $1290 \mathrm{~m} .15 .05 .2000$ \\
\hline 52: Dereçine, $1280 \mathrm{~m} .28 .05 .2000$ \\
\hline 53: Dereçine, Okkaya $1700 \mathrm{~m} .08 .07 .2000$ \\
\hline 54: Dereçine, Okkaya $1800 \mathrm{~m} .15 .07 .2000$ \\
\hline 55: Dereçine, Okkaya $1900 \mathrm{~m} .28 .07 .2001$ \\
\hline 56: Kırca Village S. $1200 \mathrm{~m} .30 .05 .1998$ \\
\hline 57: Kirca Village S. $1200 \mathrm{~m} .30 .05 .1999$ \\
\hline 58: Dort Valley $1050-1100 \mathrm{~m} .19 .05 .1999$ \\
\hline 59: Dort Valley $1100 \mathrm{~m} .29 .05 .1999$ \\
\hline 60: Dort Valley $1200 \mathrm{~m} .29 .04 .2000$ \\
\hline 61: Dort Valley $1150 \mathrm{~m} .28 .05 .2000$ \\
\hline 62: Dort Valley $1200 \mathrm{~m} .28 .05 .2000$ \\
\hline 63: Dort Valley $1200 \mathrm{~m} .10 .06 .2000$ \\
\hline 64: Dort Valley $1100 \mathrm{~m} .08 .07 .2000$ \\
\hline 65: Dort Valley $1200 \mathrm{~m} .14 .07 .2000$ \\
\hline 66: Dort Valley $1300 \mathrm{~m} .14 .07 .2000$ \\
\hline 67: Dort Valley $1100 \mathrm{~m} .05 .08 .2000$ \\
\hline 68: Dort Valley, $1200 \mathrm{~m} .19 .05 .1999$ \\
\hline 69: Dort Valley, $1250 \mathrm{~m} .29 .05 .1999$ \\
\hline 70: Dort Valley, $1300 \mathrm{~m} .29 .08 .1999$ \\
\hline 71: Dort Valley, $1280 \mathrm{~m} .28 .05 .2000$ \\
\hline 72: Dort Valley, $1300 \mathrm{~m} .10 .06 .2000$ \\
\hline 73: Deresinek Valley $1300 \mathrm{~m} .05 .08 .2000$ \\
\hline 74: Deresinek Valley $1400 \mathrm{~m} .19 .08 .2000$ \\
\hline
\end{tabular}

Carbonell (1998) identified Agrodiaetus cilicius species as a result of his study conducted in Toros Mountains and reported that its invasion reaches up to Sultandağları.

\section{MATERIALS AND METHODS}

Sultandağları is an extension of Toros Mountains toward Central Anatolia in the direction of NW-SE. Average altitude of Sultandağları rising like a wall from the plain surface with an average altitude of 970 to $980 \mathrm{~m}$ is around $2000 \mathrm{~m}$ and the altitude decreases gradually from NW toward SE until $1700 \mathrm{~m}$ around Doğanhisar. Certain high peaks on the mountain and their altitudes, from NW toward SE, are: Sultandede Peak $2311 \mathrm{~m}$, Mezarlık Peak 2274 m, Toprak Peak 2519 m, Gelincikana Peak 2610 m, Cığırgan Peak 2308 m, Demirlik Peak 2020 m, Mercansivri Peak 2276 m, Gedik Peak 2181 m, Taşpınar Plateau 2300 m, Başyurt Peak 2424 m, Batman Peak 1993 m, Tekke Mount 2169 m. As understood according to these altitudes, the mountain's altitude relative to the plain surface sometimes reaches 1000 to $1100 \mathrm{~m}$ (Atalay, 1977). Sultandağları has a climatic typology called "Central Anatolian 
Table 2. Locations and dates of collections of butterflies in Çay, Afyonkarahisar, Turkey. No Locality Altitude Collection Date

\begin{tabular}{|c|c|}
\hline 75: Çay Valley 1100 m. 01.06.1998 & \\
\hline 76: Çay Valley 1100 m. 11.06.1998 & \\
\hline 77: Çay Valley 1100 m. 05.07.1998 & \\
\hline 78: Çay Valley 1200 m. 05.07.1998 & \\
\hline 79: Çay Valley 1050 m. 09.04.1999 & \\
\hline 80: Çay Valley 1200 m. 14.04.2000 & \\
\hline 81: Çay Valley 1250 m. 15.04.2000 & \\
\hline 82: Çay Valley, Hacıpınar 1300 m. 06.05.1999 & \\
\hline 83: Çay Valley, Hacıpınar 1200 m. 20.04.2000 & \\
\hline 84: Çay Valley, Hacıpınar 1300 m. 11.06.2000 & \\
\hline 85: Çay Valley, Hacıpınar 1200 m. 29.07.2000 & \\
\hline 86: Çay Valley, Kireçlik 1400 m. 05.07.1998 & \\
\hline 87: Çay Valley, Kireçlik 1300 m. 18.05.1999 & \\
\hline 88: Çay Valley, Kireçlik 1300 m. 15.04.2000 & \\
\hline 89: Çay Valley, Kireçlik 1400 m. 13.05.2000 & \\
\hline 90: Nişankaya Location 1200 m. 04.07.1998 & \\
\hline 91: Karamık 1050 m. 20.07.1998 & \\
\hline 92: Kirazlıyayla 1400 m. 01.08.1998 & \\
\hline 93: Kirazlıyayla 1400 m. 03.07.1999 & \\
\hline 94: Kireçlik Hill 1700 m. 28.07.1998 & \\
\hline 95: Gökseki Hill 1900 m. 22.07.2000 & \\
\hline 96: Akkaya Hill 1800 m. 27.07.1999 & \\
\hline 97: Mezarlık Hill 1900 m. 09.08.1999 & \\
\hline 98: Sarıyayla Hill 1200 m. 18.05.1999 & \\
\hline 99: Sarıyayla Hill 1600 m. 17.07.1999 & \\
\hline 100: Boncuklu, Gökgedik 1900 m. 19.08.1998 & \\
\hline 101: Boncuklu, Gökgedik 1900 m. 09.08.1999 & \\
\hline 102: Boncuklu Hill 1850 m. 18.07.1998 & \\
\hline 103: Boncuklu Hill 1850 m. 01.08.1998 & \\
\hline 104: Boncuklu Hill 1700 m. 01.06.1999 & \\
\hline 105: Boncuklu Hill 1700 m. 10.07.1999 & \\
\hline 106: Boncuklu Hill 1700 m. 09.08.1999 & \\
\hline 107: Boncuklu Hill 1700 m. 22.07.2000 & \\
\hline 108: Boncuklu Hill 1800 m. 29.07.2000 & \\
\hline 109: Gölgelikaya 1600 m. 14.08.1998 & \\
\hline 110: Karıncaova 1250 m. 03.07.1999 & \\
\hline
\end{tabular}

Steppe Climate". However, local climatic variations exist between the basins of Sultandağları-Akşehir-Eber and especially, in northern and northwestern parts of Sultandağları. Thus, dry steppe climatic characteristics are prevalent in the said areas (Atalay, 1977).

This study was conducted from 1997 to 2001 in Sultandağları, Afyonkarahisar, Turkey, to identify its butterfly fauna. 110 field surveys were carried out between March to August in different habitat types and ranging in altitude from 1050 to $1900 \mathrm{~m}$ (Tables 1 and 2).

Specimens were collected using a sweep net and killed in killing jars with ethyl acetate. Each specimen was put into a labeled envelope and brought to the laboratory to be spread and dried. Identifications were based on the studies of Hesselbarth et al. (1995), Hofmann and Marktanner (1995), Tolman and Lewington (1997), and the author's reference collections. The specimens collected from the study area were taken from different stations during 110 field studies. These stations (Material Examined, Locality No) are chronologically listed in Tables 1 and 2 (Sultandağı and Çay).

\section{RESULTS AND DISCUSSION}

A total of 131 species belonging to seven families and two super families of Rhopalocera were identified. The highest number of species belongs to Lycaenidae (45, $30.4 \%$ ), followed by Satyridae (24, 18.2\%), Argynnidae (21, 16\%), Pieridae (17, 13\%), Hesperiidae (17, 13\%), Papilionidae (6, 4.6\%) and Libytheidae (1, 0.8\%). The list of species, according to Koçak and Kemal (2009), and the localities from which they were collected in Sultandağları are in Table 3.

Sultandağları Mountains has a rich flora and fauna. The different ecosystems provide multiple habitat types for animals and plants, which may increase diversity. For more thorough sampling, butterflies in Sultandağları were collected at 110 sites in different vegetation types, including agricultural fields and in forests, at altitudes ranging from 1050 to $1900 \mathrm{~m}$ and at different times. All these characteristics (locality, vegetation type, altitude, and date) affect biological distributions, especially of the butterflies in this study.

In this study, the Papilionoidea and Hesperioidea faunas in Sultandağları were comprehensively evaluated, compared with previous reports, and updated based on taxonomic and nomenclatural changes in recent years. A total of 131 species belonging to seven families of Lepidoptera were identified. These families and number of species are: Papilionidae (6); Pieridae (17); Libytheidae (1); Argynnidae (16); Satyridae (24); Lycaenidae (45) and Hesperiidae (17). Among these, Tomares nesimachus (Oberthür, 1893), is identified for the first time in Sultandağları Mountains for fauna of the Afyonkarahisar during this study. 117 species were previously known from Sultandağları, and the remaining 14 species (indicated with asterisks in Table 2) were new records for the region. In addition, Archon apollinus was also recorded by the author in Centeral Afyonkarahisar on 31 March 2001.

The larvae of some of the species identified in this study can cause economic damage to plants. Among these species Zerynthia deyrollei, Anthocharis cardamines, Euchloe ausonia, Pieris brassicae, Pieris rapae, Pontia edusa, Melanargia larissa, Vanessa cardui, Polyommatus agestis, and Polyommatus icarus have been mostly observed in agricultural areas. $P$. brassicae and $P$. rapae feed on cultivated Brassicaceae and are common pests on Brassica oleracea as well as Capparis spinosa (Capparaceae); V. cardui and $P$. icarus are widespread on Fabaceae, including Lotus, Trifolium, Astragalus, and Medicago; Lampides boeticus feeds on Prunus armeniaca and Pisum sativum (Rosaceae); Iphiclides podalirius feeds on cultivated species such as Prunus spinosa, P. armeniaca, and P. avium; and Leptidea sinapis feeds on Lotus corniculatus in Sultandağları.

Most of the species were caught in meadows and prairies because of the variety of plants and abundance 
Table 3. Systematic list of butterfly species collected in Sultandağları, Afyonkarahisar, Turkey. Localities are as in Tables 1 and 2.

Superfamily: PAPILIONOIDEA Latreille, (1802) (6 Families)

Family: PAPILIONIDAE Latreille, (1802) (6 species):

Iphiclides podalirius (Linnaeus, 1758)

Papilio (Alexanoria) alexanor (Esper, 1800)

Papilio machaon (Linnaeus, 1758)

Parnassius apollo (Linnaeus, 1758)

*Parnassius (Driopa) mnemosyne (Linnaeus, 1758)

Zerynthia (Allancastria) deyrollei (Oberthür, 1869)

Family: PIERIDAE (Duponchel, 1835) (17 species) Anthocharis cardamines (Linnaeus, 1758)

*Anthocharis gruneri (Herrich-Schäffer, 1851)

Aporia crataegi (Linnaeus, 1758)

Colias (Neocolias) aurorina Herrich-Schäffer, [1850]

Colias (Eriocolias) crocea (Fourcroy, 1785)

Colias alfacariensis (Ribber, 1905)

Euchloe ausonia (Hübner, 1804)

Gonepteryx farinosa (Zeller, 1847)

Gonepteryx rhamni (Linnaeus, 1758)

Leptidea duponcheli (Staudinger, 1871)
$87,68,14,69,57,51,19,71,72,84,33,40,41,21,85,73,74$

$24,33,41,21,65$

$1,102,98,39,99,30,19,40,41,53,65,12,95,108,73$

$5,12,54,22$

$5,26,2,48,68,14,69,38,61,71,72,63,84,33,34,40,20,9,11$

$26,58,31,14,71$

$51,47,46,2,26,48,98,87,58,68,14,80,88,60,83,89,52,19,71,3$

79

$1,6,43,25,24,77,37,7,98,14,3,69,71,72,63,84,40,32,33,34,9,20,4,11,41,53$, $64,65,54$

$1,37,36,7,20,4,41,2$

$5,1,15,24,77,45,37,7,36,26,87,68,14,3,69,59,29,99,101,60,51,47,71,72,62$ $63,84,32,33,40,34,9,4,41,64,12,65,54,53,95,13,22,18,108,85,73,67,74,23$

$5,1,24,25,77,44,37,7,36,92,51,26,2,87,68,3,14,69,29,99,89,31,61,62,71$, $63,84,20,41,11,53,64,12,66,54,95,18,22,85,67,73,74$

26, 31

$24,37,7,36,89,53,54,74$

$43,24,37,36,46,51,26,2,48,87,58,14,3,69,99,101,17,80,83,60,50,31,71,72$, $63,84,35,53,18,108,85,74$

$89,40,85$ 
Table 3. Contd.

Leptidea sinapis (Linnaeus, 1758)

Pieris brassicae (Linnaeus, 1758)

Pieris (Artogeia) ergane (Geyer, 1828)

Pieris (Artogeia) rapae (Linnaeus, 1758)

Pieris (Artogeia) mannii (Mayer, 1851)

Pieris (Artogeia) pseudorapae (Verity, 1908)

Pontia edusa (Fabricius, 1777)

Family: LIBYTHEIDAE Boisduval, 1833 (1 species):

Libythea celtis (Laicharting, 1782)

\section{Family: ARGYNNIDAE Duponchel, [1835] (21 species):}

Aglais urticae (Linnaeus, 1758)

Argynnis (Speyeria) aglaja (Linnaeus, 1758)

Argynnis (Fabriciana) niobe (Linnaeus, 1758)

Argynnis (Pandoriana) pandora [Denis and Schiffermüller], 1775)

${ }^{*}$ Boloria (Clossiana) euphrosyne (Linnaeus, 1758)

Brenthis daphne (Bergstasser, 1780)

${ }^{*}$ Brenthis hecate (Denis and Schiffermüller, 1775)

Issoria lathonia (Linnaeus, 1758)

Limenitis reducta Staudinger, 1901

* Melitaea (Mellicta) athalia (Rottemburg, 1775)

Melitaea cinxia (Linnaeus, 1758)

Melitaea (phoebe) punica Oberthür, 1876
$56,45,51,46,47,2,26,16,48,87,98,58,14,3,69,88,60,50,89,31,61,71,72,63$ $35,21,85,73$

$24,46,51,47,16,2,26,87,48,68,98,58,14,84,40,9,12,54,18,85,74,23$

$24,51,50,54$

$45,103,16,48,87,58,69,80,83,60,50,89,71,54,73$

54

$1,24,56,92,51,46,2,16,26,48,87,98,58,68,14,106,80,89,50,31,71,34,4,64$ $53,54,85,18,73,67,74$

$24,25,56,78,45,7,92,68,69,80,88,61,63,84,33,34,9,64,53,12,65,54,22,85$ $67,73,74$

17,80

$37,58,59,38,33,18$

$1,5,44,45,37,7,92,95,4,35,53,65,54,22,18,13,85,108$

$25,44,45,69,29,27,84,9,54,18,85$

$42,24,45,37,92,69,28,41,54,18,108,23$

93

$24,27,93,64,74$

110

$24,45,37,36,7,92,103,46,51,47,26,2,482,98,58,68,87,48,14,3,69,38,29,106$, $80,88,31,71,72,84,34,9,41,53,21,54,18,73$

$42,24,77,45,92,16,48,87,58,14,27,69,71,72,61,63,84,34,4,35,64,53,65,85$ $67,73,74$

$5,8,110,105,9,20,4,11,53,12,108$

$56,42,37,46,47,26,16,2,58,31,14,3,29,28,71,61,63,84,32,34,20$

$58,69,31,62,84,72$ 
Table 3. Contd

Melitaea (Didymaeformis) didyma (Esper, 1779)

Melitaea (Didymaeformis) fascelis (Fabricius, 1787)

Nymphalis antiopa (Linnaeus, 1758)

Nymphalis polychloros (Linnaeus, 1758)

Polygonia (Comma) c-album (Linnaeus, 1758)

${ }^{*}$ Polygonia (Comma) egea (Cramer, 1775)

Thaleropis ionia (Eversmann, 1851)

Vanessa atalanta (Linnaeus, 1758)

Vanessa cardui (Linnaeus, 1758)

\section{Family: SATYRIDAE Boisduval, 1833 (24 species):}

Arethusana arethusa (Denis and Schiffermüller, 1775)

Brintesia circe (Fabricius, 1755)

Chazara (Neochazara) anthe (Hoffmannsegg, 1804)

Chazara briseis (Linnaeus, 1764)

Coenonympha leander (Fabricius, 1787)

Coenonympha pamphilus (Linnaeus, 1758)

Hipparchia (Neohipparchia) fatua (Freyer, 1844)

Hipparchia (Parahipparchia) aristaeus (Bonelli, 1826)

*Hipparchia (Neohipparchia) statilinus (Hufnagel, 1766)

Hipparchia syriaca (Staudinger, 1871)

Hyponephele lupina (Costa, 1836)

Hyponephele lycaon (Rottemburg, 1775)

Kirinia roxelana (Cramer, 1777)

*Lasiommate maera (Linnaeus, 1758)

*Lasiommata megera (Linnaeus, 1767)

Maniola (Telmessiola) telmessia (Zeller, 1847)

Maniola jurtina (Linnaeus, 1758)

Melanargia (Turcargia) larissa (Geyer, 1828)
$45,5,42,77,45,37,102,59,33,9,12$

$45,5,42,77,45,37,102,59,33,9,12$

$99 ; 79 ; 82$

$37 ; 48 ; 89$

$17 ; 89 ; 50$

$97 ; 80 ; 50$

61

$45,37,14,3,80,50,72,4,11,53,18,10$

$1,42,56,24,44,45,15,25,77,37,7,92,48,17,84,40,20,4,18$

$94 ; 109$

$1,5,24,44,45,102,64$

$15,25,97,22$

$1,45,24,15,37,7,102,99,53,12,21,54,18,13,85,74,23$

$45,37,7,82,14,3,69,62,72,84,40,9,4,11$

$92,46,49,47,26,2,16,48,98,87,48,58,68,14,3,69,59,29,31,71,61,63,84,34$ $32,85,73,67,74$

$60,72,73,74$

$45,37,7,15,109,105,28,9,12$

$92 ; 109 ; 23$

$24,1,44,45,36,36,105,34,35,64,22,74,23$

$5,24,45,44,109,27,99,106,54,13$

$92,109,99,106,35,54,22,13,18,73,74$

$24,44,45,94,99,35,21,73,74$

$56,7,109,16,2,87,48,59,38,41,74$

$46,16,26,48,58,28,38,104,99,40,41$

$1,76,42,24,44,45,92,14,59,28,100,63,4,35,65,85,67$

$1,24,14,69,59,28,93,63,33,35,64,73,67,74$

$1,5,24,15,44,45,77,37,7,36,99,101,64,21,65,54,95,22,18,85,108,73$ 
Table 3. Contd.

Pseudochazara (Achazara) anthelea (Hübner, 1824)

Pseudochazara beroe (Freyer, 1843)

Pseudochazara lydia (Staudinger, 1878)

Pseudochazara mamurra (Herrich-Schäffer, 1846)

Pseudochazara mniszechii (Herrich-Schäffer , 1851)

Satyrus (Asatyrus) ferulus (Fabricius, 1793)

Family: LYCAENIDAE Stephenes, 1829 (45 species):

Callophrys rubi (Linnaeus, 1758)

Celastrina argiolus (Linnaeus, 1758)

Cupido minimus (Fuessly, 1775)

Cupido osiris (Meigen, 1829)

Glaucopsyche alexis (Poda, 1761)

Glaucopsyche (Iolana) lessei (Bernardi, 1964)

Lampides boeticu (Linnaeus, 1767)

Lycaena (Alciphronia) alciphron (Rottemburg, 1775)

Lycaena (Heodes) virgaureae (Linnaeus, 1758)

Lycaena (Loweia) tityrus (Poda, 1761)

Lycaena (Thersamonia) thersamon (Esper, 1784)

Lycaena (Thersamonia) thetis (Klug, 1834)

Lycaena phlaeas (Linnaeus, 1761)

Plebejus (Kretania) carmon (Gerhard, 1851)

Plebejus (Lycaeides) idas (Linnaeus, 1761)

Plebejus (Plebejides) sephirus (Frivaldzky, 1835)

Plebejus argus (Linnaeus,1758)

Polyommatus (Albulina) loewii (Zeller, 1847)
$24,25,44,45,37,7,36,35,65$

$96 ; 95$

$86,99,73,74,23$

$24,92,99,85,73,74$

$86,77,97,95$

$82,26,16,48,68,58,60,89,31,71,62,84$

$87,80,60,89,71,63$

$99,70,61,62,63$

$14,27,70,71,61,63,54$

$58,14,69,60,31,62,63$

$75,42,48,58$

$42,24,86,69,63,35,53,21,95,85,73,67,23,74$

$90,2,26,68,58,3,53,12,54,18,90,85,73$

$5,109,18$

$82,2,48,98,58,3,99,60,61,71,62,63,84,85,73,74$

$56,109,84,54,73,74$

$94,106,54,95,107,18,108$

$94,58,93,54,74$

$98,59,28,62,61,63,84,33,4,95$

24, 9, 54, 95

$42,86,36,87,48,59,20,4,13$

$5,1,68,7,72,53,12,54,95,18,13$

$45,63,84,34,4,65$ 
Table 3. Contd.

Polyommatus (Eumedonia) eumedon (Esper, 1780)

Polyommatus (Aricia) anteros (Freyer, 1838)

Polyommatus (Aricia) agestis (Denis and Schiffermüller, 1775)

*Polyommatus (Aricia) artaxerxes (Verity, 1936)

Polyommatus (Agrodiaetus) actis (Herrich- Schäffer , 1851) Polyommatus (Admetusia) admetus (Esper, 1783)

Polyommatus (Admetusia) ripartii (Freyer, 1830)

Polyommatus (Agrodiaetus) hopfferi (Gerhard, 1851)

*Polyommatus (Agrodiaetus) poseidon (Herrich-Schäffer, 1851)

Polyommatus (Agrodiaetus) wagneri (Forster, 1956)

Polyommatus (Agrodiaetus) iphigenia (Herrich-Schäffer, 1847)

Polyommatus (Cyaniris) bellis (Freyer, 1842)

Polyommatus (Lysandra) bellargus (Rottemburg, 1775)

Polyommatus (Lysandra) ossmar (Gerhard, 1851)

Polyommatus (Meleageria) dophnis (Denis and Schiffermüller, 1775)

Polyommatus (Plebicula) amandus (Schneider, 1792)

Polyommatus (Sublysandra) cornelius (Freyer, 1850)

Polyommatus (Thersitesia) thersites (Canterer, 1835)

Polyommatus icarus (Rottemburg, 1775)

Pseudophilotes vicrama (Moore, 1865)

Quercusia quercus (Linnaeus, 1758)

Satyrium (Nordmannia) acaciae (Fabricius, 1787)

Satyrium (Nordmannia) ilicis (Esper, 1779)

Satyrium (Strymonidia) spini (Fabricius 1787)

*Tomares (nogeli) nesimachus (Oberthür, 1893)

*Tomares (nogelii) nogelii (Freyer, 1851)
99

$51,16,48,87,58,14,59,69,29,38,61,71,63,84,4,9,85,67,74$

$42,51,47,26,48,87,58,68,14,59,69,110,89,31,61,62,63,84,34,4,9,35,64,53$, $65,85,73,74$

$45,51,46,16,87,69,31,73,67$

$25,105,106,54,95,108$

$109,35,73$

$93,105,65,21,54,95$

$109,35,65,22,85,108,73,23,74$

95

91

$105,53,65,95$

$2,26,87,68,58,3,69,29,38,31,62,63,84,4,20$

$56,43,44,7,47,51,26,16,68,48,87,98,14,59,69,29,27,99,101,31,61,71,62,63$, $84,34,4,9,20,53,73,23,74$

$94,69,99,54,85,73,74$

$45,86,109,54,85,108,73,74$

$82,16,58,14,69,27,61,63,72,84,64$

$26 ; 62 ; 73$

$53,64,12,65,54,18,13,85,73,67,23,74$

$5,1,25,56,15,37,36,51,46,26,16,48,87,98,68,14,3,59,69,29,38,99,101,31$, $61,62,71,63,84,40,33,32,34,4,20,53,64,12,65,95,22,13,18,85,73,67,23,74$

$45,46,48,58,3,99,20,21,65,67,23$

$1 ; 21$

86,95

$90,45,36,35,85$

$1,24,102,35,95$

99

$16 ; 62$ 
Table 3. Contd.

\begin{tabular}{|c|c|}
\hline Turanana endymion (Freyer, 1850) & $99 ; 22 ; 108$ \\
\hline \multicolumn{2}{|l|}{ Family: HESPERIIDAE Latreille, 1809 (17 species): } \\
\hline Carcharodus (Lavatheria) lavatherae (Esper, 1783) & $90,45,53,108,23$ \\
\hline Carcharodus alceae (Esper, 1780) & $102,69,81,89,61,64,53,12,73,74$ \\
\hline Erynnis (Hesperopegasus) marloyi (Boisduval, 1834) & $62,40,41$ \\
\hline Hesperia comma (Linnaeus, 1758) & $23 ; 54$ \\
\hline Muschampia nomas (Lederer, 1855) & $25,37,86,36,63,53,12,54,22$ \\
\hline Muschampia proteides (Wagner, 1929) & $44,109,110,99,106,21,54,85,73,23,74$ \\
\hline Ochlodes venatus (Bremer and Grey, 1852) & $5,42,24,45,99,110,72,53$ \\
\hline Pyrgus serratulae (Rambur, 1839) & $7,68,58,14,3,69,38,29,62,61,63,84,34,34,4,9$ \\
\hline Pyrgus sidae (Esper, 1784) & $27,63,84,32,4,20,12$ \\
\hline Spialia (Neaspialia) orbifer (Hübner, 1823) & $42,24,44,7,26,48,58,59,49,89,31,62,61,71,63,84,32,34,4,20,35,53,54,74$ \\
\hline Thymelicus lineolus (Ochsenheimer, 1808) & $5,1,24,25,44,45,37,36,7,59,110,63,32,20,64,53,12,54,22,18,85$ \\
\hline Thymelicus sylvestris (Poda, 1761) & $\begin{array}{l}5,1,24,45,77,44,37,37,7,36,59,99,106,84,33,4,20,35,53,64,12,21,54,22,13, \\
85,73\end{array}$ \\
\hline
\end{tabular}

of flowers there. The greatest diversity of butterfly species was observed in spring and summer seasons, which provide the most food resources for butterfly larvae and adults and have adequate temperatures for development.
The most widespread species in the study area were: I. podalirius, Papilio machaon, Parnassius mnemosyne, A. cardamines, Aporia crataegi, Colias crocea, E. ausonia, Gonepteryx rhamni, L. sinapis $P$. brassicae, $P$. rapae, $P$. pseudorapae,
P. edusa, Argynnis aglaja, A. pandora, Issoria lathonia, Limenitis reducta, Melitaea cinxia, V. cardui, Chazara briseis, Coenonympha leander, C. pamphilus, Hipparchia syriaca, Hyponephele lycaon, Lasiommata maera, L. megera, Maniol 
telmessia, M. jurtina, M. larissa, Callophrys rubi, L. boeticus, Lycaena alciphron, L. tityrus, Polyommatus anteros, $P$. agestis, $P$. bellis, $P$. bellargus, $P$. thersites, $P$. icarus, Carcharodus lavatherae, C. alceae, Erynnis tages, Muschampia proteide, Pyrgus melotis, $P$. serratulae, Spialia orbifer, Thymelicus lineolus, and $T$. sylvestris.

The Papilionoidea and Hesperioidea fauna of the Sultandağları Mountains was investigated in detail with this study and results of the studies conducted on the subject were also evaluated together. Furthermore; recent changes on Lepidoptera taxonomy and nomenclature were taken into consideration in this study and thus scientific names of fauna in this region were updated. 14 taxa of Papilionoidea and Hesperioidea in total have been identified until now from Sultandağları. Their distributions according to families are as: Papilionidae (1), Argynnidae (2), Satyridae (1), Lycaenidae (7) and Hesperiidae (3). According to the recent taxonomic studies (Hesselbarth et al., 1995), only five names from the proposed taxons still remains valid today. Others are mentioned in the literature as just young synonym names. The list relating to these names is given below. The 14 valid taxa, which have been identified in Sultandağları are not endemic for the area covered in this study because they exist in other regions of Turkey and even in some neighbor countries. Papilionidae Parnassius apollo anatolicus Pagenstecher, 1912 , today is accepted as junior synonym of Parnassius apollo (Linnaeus, 1758).

Argynnidae: Mellicta athalia anatolica Wagner, 1929, today is accepted as junior synonym of Melitaea (Mellicta) athalia (Rottemburg, 1775). Brenthis daphne anatolica Belter, 1935, today is accepted as junior synonym of Brenthis daphne (Bergstasser, 1780).

Satyridae: Eumenis statilinus minutula Verity, 1938, today is accepted as junior synonym of Hipparchia (Neohipparchia) statilinus (Hufnagel, 1766).

Lycaenidae: Strymon lynceus ssp. anatolicus Lattin, 1950, today is accepted as junior synonym of Satyrium (Strymonidia) spini (Fabricius 1787). Callophrys rubi ssp. herculeana Pfeiffer, 1927, today is accepted as junior synonym of $C$. rubi (Linnaeus, 1758). Cupido (Tiora) sebrus majuspunctata Verity, 1934, today is accepted as Cupido osiris (Meigen, [1829]). Plebejus sephyrus modica Verity, 1935, today is accepted as junior synonym of Plebejus (Plebejides) sephirus (Frivaldzky, 1835). Lycaena argus ssp. sultana Forster, 1936, today is accepted as junior synonym of Plebejus argus (Linnaeus, 1758). Agrodiaetus admetus ssp. anatoliensis Forster, 1960, today is accepted as Polyommatus (Admetusia) admetus (Esper, [1783]). Agrodiaetus damone ssp. wagneri Forster, 1956, today is accepted as Polyommatus (Agrodiaetus) wagneri (Forster, 1956).

Hesperiidae: Carcharodus orientalis ssp. centralanatolica Pfeiffer, 1927, today is accepted as junior synonym of Carcharodus (Reverdinus) orientalis Reverdin, 1913.
Hesperia proto proteides Wagner, 1929, today is accepted as Muschampia proteides (Wagner, 1929). Hesperia persica postranae Pfeiffer, 1927, is accepted as junior synonym of Pyrgus armoricanus (Oberthür, 1910).

This study contributed to our knowledge of the lepidopteran fauna of Sultandağları in Afyonkarahisar province, Turkey. Scientific and taxonomic studies from this region are very limited in number. New studies of insect distributions should be carried out before agricultural and forest ecosystems are destroyed. In addition, the continuing illegal use of forests and incorrect land-use practices threaten to destroy biodiversity in the Aegean region. Therefore, detailed studies should be carried out as quickly and accurately as possible to evaluate the biological diversity in Turkey.

\section{REFERENCES}

Atalay İ (1977). Sultandağları ile Akşehir ve Eber gölleri havzalarının strüktüral, jeomorfolojik ve toprak erozyonu etüdü. Atatürk Üniv. Yay. 500. Erzurum.

Belter G (1935). Zwei neue Brenthis-Rassen aus Kleinasien. Ent. Z. Frankf. M. 49:71-72.

Carbonell F (1998). Contribution a la connaissance du genre Agrodiaetus Hübner, 1822 Agrodiaetus cilicius n. sp. en Turquie méridionale (Lepidoptera, Lycaenidae). Linneana Belgica 16(8):343349.

Forster W (1936). Beitrag zur Systematik des Tribus Lycaenini unter besonderer Berücksichtigung der argyrognomon- und der argusGruppe. Mitt Münch Ent. Ges. 26(2):41-140.

Forster W (1956). Bausteine zur Kenntnis der Gattung Agodiaetus Scudd. (Lep., Lycaen.) I. Z. Wien Ent. Ges. 41:42-61.

Forster W (1960). Einige neue formen der Gattung Agrodiaetus Scudd.(Lep., Lycaen.). Ent. Z. Frankf. M. 70(3):17-22.

Forster W (1961). Bausteine zur kenntnis der Gattung Agodiaetus Scudd. (Lep., Lycaen.) II. Z. Wien Ent. Ges. 46:8-94.

Hesselbarth G, Van Oorschot H, Wagener S (1995). Die Tagfalter der Turkei, 3 band. Selbstverlag Sigbert Wagener Hemdener Weg 19, D 46399 Bocholt, Germany.

Hofmann H, Marktanner T (1995). Butterflies and moths of Britain \& Europe. Collins Nature Guides. Harper Collins, London.

Koçak AÖ, Seven S (1994). Türkiye Lepidoptera Bibliyografyası. Cent. Ent. Stud. Memoirs. 2:1-141.

Koçak AÖ, Kemal M (2009). Revised checklist of the Lepidoptera of Turkey, Cent. Ent. Stud. Priamus Supplement 17:1-253.

Lattin G de (1950). Türkische Lepidopteren-I. Ist Univ. Fen Fak Mec. (Ser b). 15 (4):301-328.

Pfeiffer E (1926). Ein Beitrag zur Insectenfauna von Anatoluen. Mitt. Münch. Ent. Ges. 16:99-110.

Schurian KG, Rose K (1991). Contribution to the knowledge of Polyommatus (Aricia) hyacinthus (Herrich-Schäffer, 1847) and the description of a new subspecies (Lepidoptera, Lycaenidae). Linneana Belg. 13(2):80-93.

Schwingenschuss L (1938-1939). Sechster Beitrag zur Lepidopteren fauna Inner-Anatoliens. Ent. Rdsch., 55:141-700.

Schwingenschuss L (1939). Sechster beitrag zur Lepidopteren fauna Inner-Anatoliens. Ent. Rdsch. 56:133-252.

Tolman T, Lewington R (1997). Butterflies of Britain \& Europe. Collins field guide. Harper Collins, London. (The year of publication do not correspond with that cited in the article)

Verity $R$ (1934). The lowland races of butterflies of the Upper Rhone Valley. Entomologist's Rec. J. Var. 46:1-40.

Verity R (1935). Races de Lysandra thersites (Lep.,Cant.) Champ. et de Plebeius sephyrus Friv. de I' Asie Mineure. Bull. Soc. Ent. Fr. 40:243245.

Verity $R$ (1936). The butterfly races of Macedonia. Entomologist's Rec. J. Var. 48:1-8. 
Verity R (1937). The butterfly races of Macedonia. Entomologist's Rec. J. Var. 49:9-22.

Verity R (1937). Variation of some butterflies in Anterior Asia and in Morocco. Entomologist's Rec. J. Var. 49(11):1-4.

Verity R (1938). Variation of some butterflies in Anterior Asia and in Morocco. Entomologist's Rec. J. Var. 50(5):9-12.

Wagner F (1929). Weitrer Beitrag zur Lepidopterenfauna InnerAnatoliens. Mitt. Münch. Ent. Ges. 19:1-28, 57-80. 